\title{
GLOBAL WIRELESS E-VOTING SYSTEM
}

\author{
Devireddy Mahendra Reddy ${ }^{1}$, A K Sravya Reddy ${ }^{2}$ \\ ${ }^{I}$ B-Tech, Computer Science and Engineering, Sree Vidyanikethan Engineering College, Andhra Pradesh, India \\ ${ }^{2}$ B-Tech, Computer Science and Engineering, Sree Vidyanikethan Engineering College, Andhra Pradesh, India
}

\begin{abstract}
As we are seeing much better growth in technology but we don't see that its level is being properly utilized in the voting system. The present voting system is highly unsecured and it's not efficient in utilizing the current technology i.e., It can't determine that the person who come for voting is eligible or not, it just depends on the voting in-charge officer in the booth. Here there is also a possibility to boost the vote number as the vote count lies within the piece of equipment and if the in-charge officer is corrupted, he has the chance to do it, even while transporting the machines to the strong room. Hence we can't rely on it any more. In the projected system, i.e., "Global wireless e-voting system", machine is made smart that it can find out whether the voter is qualified for voting or not with the help of scanning the eye pattern of the voter and also the vote count is not maintained in the machine itself. Vote count is made to be stored in a remote server by converting them into radio waves. Hence there won't be any scope of escalating the vote count. Even the machine fails; there won't be any problem to the votes that are casted as they are saved in the server. By this we can reduce many problems regarding the present EVM's.
\end{abstract}

Keywords: Technology, Secured, Efficient, Retina Pattern, Remote Server Etc.

\section{INTRODUCTION}

India is a Secular, Socialist, Democratic Republic and the largest democracy in the World. Having a constitutional democracy with a parliamentary system of government, and at the centre of the system lays a obligation to hold standard, free and fair elections. The body of voters exceeds 605 million; voting in nearly 800,000 polling booths, extend across extensively varying geographic and climatic zones. Even in the snow-clad mountains in the Himalayas, the deserts of the Rajasthan and in meagerly populated islands in the Indian Ocean also polling stations are available. In democratic countries, voting plays a vital role. Hence we should adopt a perfect voting system, which should be efficient and secured. People now days need more comfort and they need things to be done so easily. They don't want to go to their respective constituency to cast their vote. They want everything to be done without troubling their comfort. This is also one of the reasons for low polling percentages. Hence with the help of current technology, we can meet their meet their requirements by allowing them to cast their vote in hands with their mobile phone. By this we can also increase the voting strength. To provide solution to all these hurdles we propose "Global Wireless E-Voting System".

\section{EVM SYSTEM AND CONVENTIONAL SYSTEM}

\subsection{Ballot Voting System}

We used to have a Ballot voting system in the past and it is also being followed in some of the very few places at present. People were provided with a ballot paper containing the list of candidates, their respective party names and symbols. All the voters had to do,was to put a swastika symbol of the candidate whom they want to elect. It had many disadvantages. This system was not a secured one. It was also not a cost effective one.

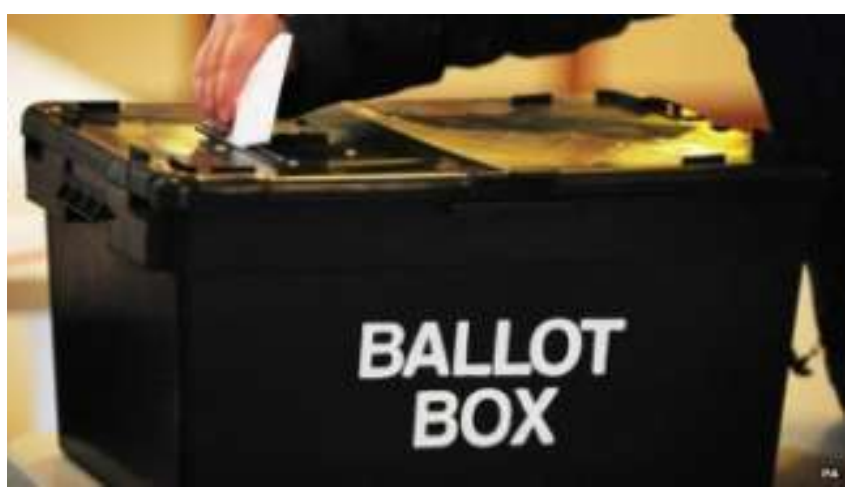

Fig-1: Ballot box

\subsection{Electronic Voting Machine (EVM)}

Now the present system is the Electronic Voting Machine (EVM). This system contains a balloting unit and a control unit. With the help of balloting we can cast our vote by pressing the symbol which lies besides the name of the candidate. Control unit stores all the votes in itself. After the completion of the voting the control unit is detached from the balloting unit. After that nobody can cast their vote and also any one can't increase the vote count. Booth in-charge officer is the one who checks the eligibility of the person who comes to vote and send them towards the machine to cast the vote. The voter has to press the button near the symbol of the candidate whom they want to elect. For getting the result, there will be a button called Result. If we press those buttons we can get the results of the election. Works are going on to introduce "Voter verified paper audit trial". In this the voter will also be given a printed paper containing the details of the vote that he has casted. Government of India has experimented this kind of voting in many places like Chennai central, Bangalore south and Mizoram. 


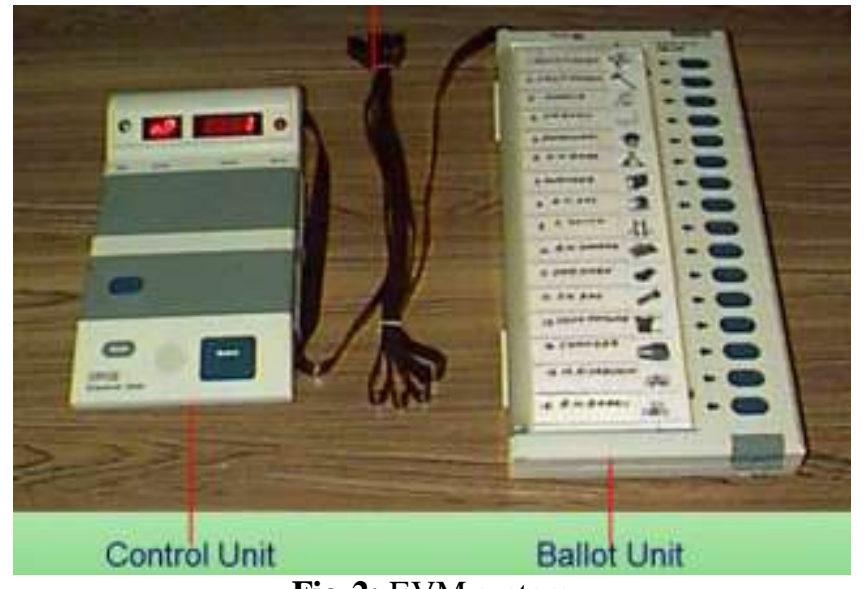

Fig-2: EVM system

\subsection{DISADVANTAgeS OF THE PRESENT}

\section{SYSTEM}

In the present system, as the votes casted will be stored in the machine itself, if the machine gets damaged the votes stored till then will be lost. The machine will not check for the eligibility of the candidate, as it was not designed to that. Voting in-charge officer will take care of those kinds of issues. If the officer is a corrupted one, he may increase the count of voting and he may also destroy the machine. Moreover this system is expensive as we need officers, secured place for counting and for election to be carried out. It needs nearly 5 million man power to conduct voting in India. The voter can't vote from the place where he is. He has to go to his respective constituency to cast his vote.

\section{PROPOSED SYSTEM}

In the Global wireless e-voting machine, the votes being casted will be stored in another remote secured server. An electronic system is used to enable the voter to vote and this vote will be transferred to the remote secured system by converting it into the radio waves. Unlike the previous systems, our system is capable of checking the legibility of the person who comes to voting through scanning his retina pattern. Even if the machine gets damaged the count of voting will not be lost and we can vote from anywhere. A person will be able to vote from mobile system or even through the internet only if he has retina scanner.

\subsection{Detailed Diagram of the Election Process}

In the proposed system, it is a machine which produces dissimilar voltages for various kinds of votes and these voltages are given as input to the Analog to Digital Converter (ADC), which converts those analog signals to digital bits then they can be converted to radio waves. Then those waves are made to reach the mobile towers and are allowed to go to the remote server.

\subsection{Interface Device}

This is an electronic device which converts the input digital signals such as (retina pattern votes and secure bits) to radio waves.

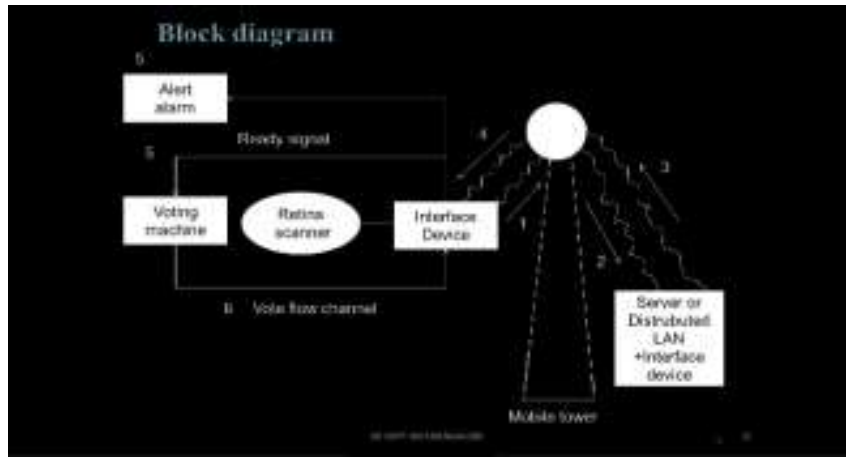

Fig-3: Interface device

Explanation of the numbers in the above diagram is as follows:

1. Radio waves are being transmitted to the mobile tower. Those waves consist of scanned retina pattern and the vote.

2. Radio waves are being transmitted from the mobile tower to the remote server.

3. Positive or negative acknowledgement is being transmitted from the server to mobile tower.

4. Positive or negative acknowledgement is being transmitted from mobile tower to Interface device.

5. Ready signal will be generated if the retina is scanned appropriately to voting machine. If not so, negative signal will be generated then alert alarm will be activated.

6. Acknowledged vote is prepared to rivulet to the interface device.

\subsection{Scanning of Eye Retina}

The eye retina scanner is an electronic device that scans the voter's eye retina pattern. The captured figure has to be converted in to the structure of a matrix where it comprises of pixels, where each pixel corresponds to a 24-bit (RGB, $8+8+8$ format)

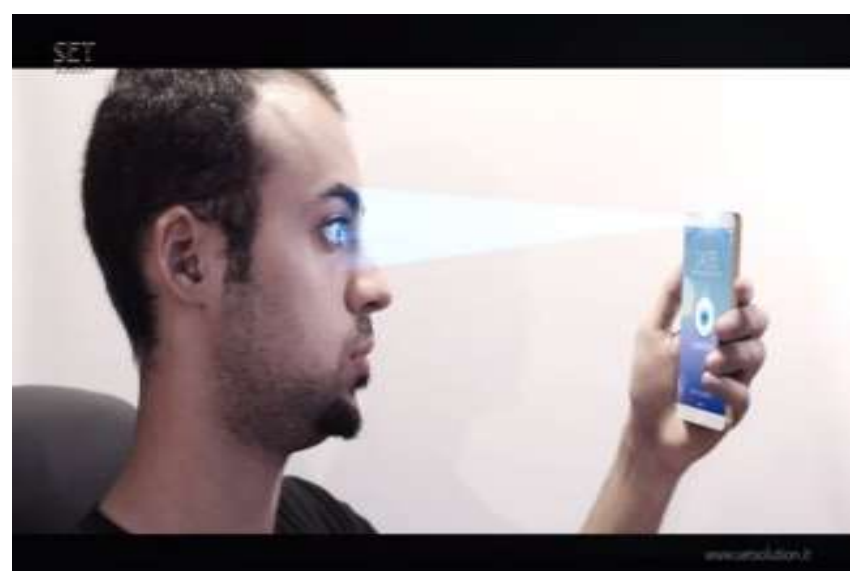

Fig-4: Retina Scanning

\section{4: Working of the Whole System}

In the global wireless e-voting system there will be two possibilities of voting. 
3.4.1. Voter can cast his vote with his mobile device, wherever he wants. But the mobile device should have internet connection and also a retina scanner.

3.4.2. Coming to another process, voter has to go to the voting booth, to cast his vote. Subsistence of this process is because every voter won't have a mobile device with internet connection and even if he has Internet connection he should also must have a retina scanner. Hence to help them and also to allow the uneducated voters to cast their votes, we go for this process. In this process, when a voter enters the voting booth, he is asked to scan his retina pattern with the help of retina scanner. Once the scanning was confirmed then the machine sends a signal to the voting machine and then the voting machine is turned on, until then it will be turned off. When the voting machine is turned on, he is allowed to cast his vote. Once casting of the vote is completed, the total information together with the scanned retina pattern and the vote is sent to the interfacing device which converts them into radio waves of mobile frequency range and these are send to cellular phone tower and then to the remote server, where the validation of these details will be done. Database consists of identification details of the voter. Once the details are authenticated and if found that the details are valid, then a positive acknowledgement is send to the voter at the voting machine telling him that the vote he casted is valid and it is counted. There will be count variable which will be incremented to one, if the voter votes for the first time. If the voter tries to vote for the second time or the details of the voter are not valid then it sends a negative acknowledgement, which will rings an alarm system .With that alarm system the voting in-charge officer will take necessary actions on the fraud voters. In this way the total voting process goes on.

\section{HURDLES IN THE PATH OF IMPLEMENTATION}

We have found that this method of voting is really time efficient and reduces the efforts of the voter. But coming to the process of implementation of it, we may encounter some problems related to

\subsection{Security}

\subsection{Efficiency}

\subsection{Geographical Problems}

The clear explanation of these is as follows:

\subsection{Security}

When the voter casts his vote, the vote and the retina pattern together has to be converted into the radio waves and then it has to be transmitted to the server. But in the process of transmission, there may be problems like signals being trapped by an attacker and he can reveal the status of the vote. As the votes will be saved in the databases, Attackers can crack the security levels of the database and can know the results of the voting. If possible he can also change the count of the votes polled. To avoid these type of problems, we adopt secured and complex encryption algorithms at the sending side and the respective decryption algorithms at the receiving side. We are also required to properly maintain security for the databases so that the attacker can't crack the database. For this we use an encryption algorithm. The encryption algorithm can be termed as Key Complex Algorithm, which is as follows,

- Take a string which you want to provide encryption.

- Determine the string length and produce random numbers equivalent to the string size.

- Generate the ASCII values for that string.

- Add the randomly generated numbers with the respective ASCII values.

- Generate the ASCII characters for those added values and the randomly generated numbers; place them alternatively.

\section{Example:}

Let the given word be: MAHEN

The given string has a length of 5 . Hence generate 5 random numbers. The numbers are:

A) 3335374043

The ASCII values for MAHEN are

$\begin{array}{lllll}\text { M A } & \mathbf{H} & \mathbf{E} & \mathbf{N}\end{array}$

B) 7765726978

Add equivalent $\mathrm{A}$ ) and $\mathrm{B}$ ) values as

$33+77,35+65,37+72,40+69,43+78$

$\begin{array}{lllll}110 & 100 & 109 & 109 & 121\end{array}$

The parallel ASCII characters for the above are:

n $\quad \mathbf{d} \quad \mathbf{m} \quad \mathbf{m} \quad \mathbf{y}$

The below are the ASCII characters for those respective random values:

! \# \% $\quad(+$

Finally encrypted data as

n ! d \# m \% m ( $\mathbf{y}+$

The ultimate encrypted information is shaped in such a way that the unsystematic information at the even places and the remaining at odd places. This is done to make the decryption much uncomplicated. The encrypted data can be decrypted simply by taking away the characters at even position from odd position character. In this way the algorithm can be implemented.

\subsection{Efficiency}

During the voting process many people will be accessing the system. So the votes being casted by the voters will be in large number going to the server. This may setback the voting process. The votes which are being acknowledged at the server is in various access mode. This is because more than one person can cast their votes at the same time from various places. If we reduce the data size then this problem may reduce. We may also apply compression algorithms in order to overcome this problem. This will decrease the data transfer. Compression methods like jpeg, gif, png etc. may be used.

We may also use distributed computing. Using distributed operating system with multiple servers is more effective than using single server. Concurrency problem in using the multiple servers can be reduced by using CDMA technique where the key values are orthogonal to each other i.e. 
$\mathrm{x} 1 * \mathrm{x} 2=0$ and $\mathrm{x} 1 * \mathrm{x} 1=1$ i.e. if anyone tries to decode the data with any other key the data will be missing as the data will be in the type $\mathrm{y} 1 * \mathrm{x} 1$.If anyone try to decode with $\mathrm{x} 2$ then effect will be as $\mathrm{y} 1 * \mathrm{x} 1 * \mathrm{x} 2=0$. This will fade away the data. And if correct decoding key i.e., $\mathrm{x} 1$ is used then decoding will be $\mathrm{y} 1{ }^{*} \mathrm{x} 1{ }^{*} \mathrm{x} 1=\mathrm{y} 1$. This decrypts the information accurately. As per the scheming concurrency for multiple access the information from all the nodes is acknowledged as $\mathrm{x} 1 * \mathrm{y} 1+\mathrm{x} 2 * \mathrm{y} 2+\mathrm{x} 3 * \mathrm{y} 3+\mathrm{x} 4 * \mathrm{y} 4$. If anyone needs information respective to the second node then simply multiply the complete equation with $\mathrm{x} 2$. This will result $\mathrm{y} 2$ as $(x 1 * y 1+x 2 * y 2+x 3 * y 3+x 4 * y 4) * x 2=y 2$. It can be seen that numeral nodes are permitted to send the data, while the server will acknowledge all the information and which ever has to be unconcerned will be multiplied with respective key.

\subsection{Geographical Troubles}

There will be few areas where we can't find internet services and mobile tower facilities .In such areas people will have to face the difficulties in casting their vote. In order to the overcome their problem it can be made to translate the casted vote and the eye retina pattern of the voter into electrical signals till the area where the appropriate facilities are found. If there is only internet service available in the area then the electronic signals generated has to be converted into the digital signals and then the votes will be casted. There is also an advantage with the system. We have many people being settled in other countries. Their vote should also be casted and therefore this can be done but the system through the mobiles itself even from there.

\section{FUTURE ENRICHMENTS}

The project can be enhanced to work in mobiles through SMS. With this method we can increase the percentage of voting. But for its implementation security becomes a problem which has to be overcome with the proper secured methods. Here when the voter casts his vote we can provide the voter with a printed paper containing the details of the vote that he has casted. With this printed paper we can reduce the fraudulent actions that can takes place.

\section{CONCLUSION}

This process of voting can be done at any place. The machine which we will be going to use in this process will provide higher level of security, authentication, reliability, and corruption-free mechanism. By this we can get the result within minutes after the completion of voting. Smallest amount human resource deployment takes place. It also saves huge amount of materials like papers etc. Hence with the utilization of this process we can increase the polling percentage with the reliable, error free, secured and efficient voting

\section{REFERENCES}

[1].https://en.wikipedia.org/wiki/Voterverified_paper_audit_ trail.
[2].http://www.ifet.ac.in/pages/intsymp14/TechnoVision\%2 0'14/papers/IT/IT02.pdf

[3]. https://en.wikipedia.org/wiki/Elections_in_India.

[4]. https://en.wikipedia.org/wiki/Indian_voting_machines

[5]. http://www.seminarsonly.com/Labels/Global-WirelessE-Voting-PDF-Abstract.php

\section{BIOGRAPHIES}

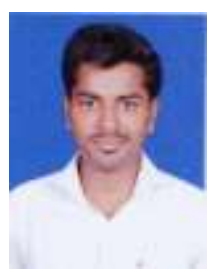

I am Mahendra Devireddy. Presently I am persuading my under graduation in Computer Science and Technology in Sree Vidyanikethan Engineering College, Tirupati.

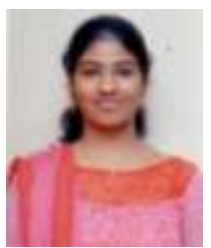

I am Agasthyareddy Kasthuri Sravya Reddy. Presently I am persuading my under graduation in Computer Science and Technology in Sree Vidyanikethan Engineering College, Tirupati. 